\title{
Pre-transitional disk nature of the AB Aur disk ${ }^{1}$
}

\author{
M. Honda ${ }^{2}$, A. K. Inoue 3 , Y. K. Okamoto ${ }^{4}$, H. Kataza ${ }^{5}$, M. Fukagawa ${ }^{6}$, T. Yamashita ${ }^{7}$, T.

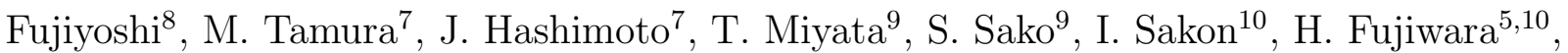 \\ T. Kamizuka ${ }^{5,10}$ and T. Onaka ${ }^{10}$
}

\begin{abstract}
The disk around $\mathrm{AB}$ Aur was imaged and resolved at $24.6 \mu \mathrm{m}$ using the Cooled Mid-Infrared Camera and Spectrometer on the $8.2 \mathrm{~m}$ Subaru Telescope. The gaussian full-width at half-maximum of the source size is estimated to be $90 \pm 6$ $\mathrm{AU}$, indicating that the disk extends further out at $24.6 \mu \mathrm{m}$ than at shorter wavelengths. In order to interpret the extended $24.6 \mu \mathrm{m}$ image, we consider a disk with a reduced surface density within a boundary radius $R_{c}$, which is motivated by radio observations that suggest a reduced inner region within about $100 \mathrm{AU}$ from the star. Introducing the surface density reduction factor $f_{c}$ for the inner disk, we determine that the best match with the observed radial intensity profile at $24.6 \mu \mathrm{m}$ is achieved with $R_{c}=88 \mathrm{AU}$ and $f_{c}=0.01$. We suggest that the extended
\end{abstract}

\footnotetext{
${ }^{1}$ Based on data collected at Subaru Telescope, which is operated by the National Astronomical Observatory of Japan.

${ }^{2}$ Department of Information Sciences, Kanagawa University, 2946 Tsuchiya, Hiratsuka, Kanagawa, 2591293, Japan

${ }^{3}$ College of General Education, Osaka Sangyo University, 3-1-1, Nakagaito, Daito, Osaka 574-8530, Japan

${ }^{4}$ Institute of Astrophysics and Planetary Sciences, Faculty of Science, Ibaraki University, 2-1-1 Bunkyo, Mito, Ibaraki 310-8512, Japan

${ }^{5}$ Department of Infrared Astrophysics Institute of Space and Astronautical Science (ISAS) Japan Aerospace Exploration Agency (JAXA) 3-1-1 Yoshinodai, Chuo-ku, Sagamihara, Kanagawa 252-5210, Japan

${ }^{6}$ Department of Earth and Space Science, Graduate School of Science, Osaka University, 1-1 Machikaneyama, Toyonaka, Osaka 560-0043, Japan

${ }^{7}$ National Astronomical Observatory of Japan, 2-21-1 Osawa, Mitaka, Tokyo 181-8588, Japan

${ }^{8}$ Subaru Telescope, National Astronomical Observatory of Japan, 650 North A'ohoku Place, Hilo, Hawaii 96720, U.S.A.

${ }^{9}$ Institute of Astronomy, School of Science, University of Tokyo, 2-21-1 Osawa, Mitaka, Tokyo 181-0015, Japan

${ }^{10}$ Department of Astronomy, School of Science, University of Tokyo, Bunkyo-ku, Tokyo 113-0033, Japan
} 
emission at $24.6 \mu \mathrm{m}$ is due to the enhanced emission from a wall-like structure at the boundary radius (the inner edge of the outer disk), which is caused by a jump in the surface density at $R_{c}$. Such reduced inner disk and geometrically thick outer disk structure can also explain the more point-like nature at shorter wavelengths. We also note that this disk geometry is qualitatively similar to a pre-transitional disk, suggesting that the AB Aur disk is in a pre-transitional disk phase.

Subject headings: protoplanetary disks — circumstellar matter — stars: pre-main sequence

\section{Introduction}

The evolution of protoplanetary disks has attracted attention because it is presumably a site of the on-going planet formation. Mostly by analyzing spectral energy distributions (SEDs), many transitional disks whose inner region is devoid of small dust grains have been detected. Recently, Espaillat et al. (2007) introduced a new sub-class called pre-transitional disks with definite near-infrared (NIR) excesses that indicate the presence of an inner disk separated from an optically thick outer disk. They suggested that such a disk is forming a gap and when the inner disk dissipates, it will become a transitional disk with an inner cavity. In both cases, the presence of a wall-like structure on the inner edge of the outer disk can explain the excess mid-infrared (MIR) emission seen in the SED. For intermediate-mass young stars such as Herbig Ae/Be stars, some objects such as HD 135344B (Grady et al. 2009) and HD 100546 (Benisty et al. 2010) are thought to be in the pre-transitional or transitional disk phase.

AB Aur (d=144-17 pc; A0 Ve, van den Ancker et al. (1997)) has been regarded as a prototypical Herbig Ae/Be star and is one of the best-studied Herbig Ae stars, with a mass of $2.4 \pm 0.2 \mathrm{M}_{\odot}$ and an age of $4 \pm 1 \mathrm{Myr}$ (DeWarf et al. 2003). Previous observations at various wavelengths revealed complex circumstellar structures at various scales. Optical and NIR coronagraphic direct images showed an extended circumstellar nebulosity of the envelope and spiral structures in the disk in scattered light (Grady et al. 1999; Fukagawa et al. 2004). Recent coronagraphic polarimetric imaging observations revealed a dust annulus at a radius of 100AU (Oppenheimer et al. 2008; Perrin et al. 2009; Hashimoto et al. 2010) with a possible fluctuation in the azimuthal direction. Millimeter and sub-mm interferometric observations showed that the gas and dust have been depleted in the central region $(r<100$ AU) of the disk (Piétu et al. 2005; Lin et al. 2006). 
At MIR wavelengths, many direct imaging and interferometric studies have been performed toward AB Aur. Chen \& Jura (2003) imaged AB Aur at 11.7 and $18.7 \mu \mathrm{m}$ using the Keck I telescope, and resolved it at $18.7 \mu \mathrm{m}$. They found that models with a disk surrounded by an envelope fit their data better than flared disk models. Applying a deconvolution technique to the $20.5 \mu \mathrm{m}$ data obtained at the $3.6 \mathrm{~m}$ Canada-France-Hawaii-Telescope (CFHT), Pantin et al. (2005) discovered a ring-like structure at 280 AU. Mariñas et al. (2006) presented 11.6 and $18.5 \mu \mathrm{m}$ images using the Gemini North telescope; however, they could not

find the ringlike structure reported by Pantin et al. (2005). They derived the intrinsic source full-width half-maximum (FWHM) to be $17 \pm 4 \mathrm{AU}$ at $11.6 \mu \mathrm{m}$ and $22 \pm 5 \mathrm{AU}$ at $18.5 \mu \mathrm{m}$, assuming a gaussian brightness distribution. Liu et al. (2005, 2007) also resolved the disk at $10.3 \mu \mathrm{m}$ by nulling interferometric observations and derived the diameter of the disk to be 24-30 AU.

In this paper, we present results of the first imaging observations of AB Aur at $24.6 \mu \mathrm{m}$ using the $8.2 \mathrm{~m}$ Subaru telescope. At $24.6 \mu \mathrm{m}$, the point-spread function (PSF) is stable compared to that at shorter wavelengths because of the larger Fried length, which enables us to observe small extended structures with high reliability. In addition, it allows us to trace the cooler part of the disk. By constructing a simple model, we discuss the disk structure of AB Aur as well as implications for the nature of the AB Aur disk.

\section{Observations and Data Reduction}

We made imaging observations with the Cooled Mid-Infrared Camera and Spectrometer (COMICS; Kataza et al. 2000; Okamoto et al. 2003; Sako et al. 2003) on the $8.2 \mathrm{~m}$ Subaru Telescope on Mauna Kea, Hawaii on 2003 October 12. AB Aur was observed using the Q24.5-OLD filter $\left(\lambda_{\nu_{\text {mean }}}=24.6 \mu \mathrm{m}, \Delta \lambda=1.9 \mu \mathrm{m}\right)$. The plate-scale of COMICS is 0.13 arcsec per pixel. The chopping throw was 10 arcsec and the position angle (PA) of the chopping direction was 30 degree. The chopping frequency was $0.45 \mathrm{~Hz}$, and the total integration time was 2005 s. Just before and after AB Aur observations, we observed $\alpha$ Tau as the PSF reference and photometric standard star. The total integration times were $401 \mathrm{~s}$ and $251 \mathrm{~s}$, respectively.

For data reduction, we employed a shift-and-add method to improve the spatial resolution. The imaging data consist of $0.983 \mathrm{~s}$ on-source integration frames. First, the thermal background and the dark current signals were removed through the subtraction of the chopped pair frames. The object was sufficiently bright to be recognized even in $0.983 \mathrm{~s}$ integration chop-subtracted frames, so we searched for the peak position by fitting a Gaussian with the source profile. Then we shifted the frames (in order to align the peak position) and 
summed them, but we excluded the frames whose gaussian FWHM deviated more than $1 \sigma$ from the mean value. This rejection of lower quality data resulted in an effective integration time of $1333 \mathrm{~s}$ for AB Aur. The same procedure was applied to the data of $\alpha$ Tau taken before and after AB Aur observations, and the effective integration times were reduced to $327 \mathrm{~s}$ and $209 \mathrm{~s}$, respectively. Flux calibration was performed using the $\alpha$ Tau template spectrum provided by Engelke et al. (2006). Standard aperture photometry was performed and the resultant flux density of AB Aur was $43.0 \pm 3.4 \mathrm{Jy}$.

\section{Results}

\subsection{Source size of AB Aur at $24.6 \mu \mathrm{m}$}

The $24.6 \mu \mathrm{m}$ images of AB Aur and $\alpha$ Tau are shown in Fig.1. It is clear that AB Aur is extended beyond the PSF at this wavelength. The disk emission appears symmetric and we could not find clear evidence of the radial profile depending on the azimuthal angle within the uncertainty. This is probably because the disk around AB Aur is nearly faceon (inclination $\sim 30^{\circ}$; Fukagawa et al. 2004). The FWHMs of shift-and-added images of AB Aur, as well as $\alpha$ Tau before and after observations of AB Aur were $0.82 " \pm 0.03 "$, $0.647 " \pm 0.007 "$ and $0.644 " \pm 0.004 "$, respectively. This error is the standard deviation of the selected frames. The FWHM of the PSF reference ( $\alpha$ Tau) is comparable to the predicted value of the diffraction-limited performance of the telescope at $24.6 \mu \mathrm{m}$. We estimate a rough source size of AB Aur by comparing the observed AB Aur image with a circular gaussian image convolved with the observed PSF image. The best-match gaussian FWHM is $90 \pm 6$ AU. This value is larger than that at shorter wavelengths, since the gaussian FWHMs of the

AB Aur disk are repoted to be $17 \pm 4 \mathrm{AU}$ at $11.6 \mu \mathrm{m}$ and $22 \pm 5 \mathrm{AU}$ at $18.5 \mu \mathrm{m}$ (Mariñas et al. 2006).

\subsection{Comparison with a simple model}

Gaussian brightness distributions are used for the sake of simplicity and they are not a good representation of the true intrinsic radial brightness profile of the disk. Thus, it would be useful to compare the observed AB Aur image with a disk model for the investigation of physical properties of the disk. We can calculate the model brightness distribution of a disk if the temperature and density structure of the disk is given. Here we derive the disk structure based on a $1+1 \mathrm{D}$ radiative transfer model similar to that in Dullemond et al. (2002); the stellar radiation transfer is solved by the so-called grazing angle approximation, 
but the radiative transfer of the dust thermal radiation is solved exactly along the disk vertical axis with a variable Eddington factor method. The dust and gas are assumed to be thermally and dynamically coupled, and the vertical density structure is determined by the hydrostatic equilibrium that is maintained consistent with the temperature structure. Scattering is not taken into account. When the disk is optically thick, the model predicts a flared geometry and reproduces the spectrum of protoplanetary disks. When the disk becomes optically thin, the grazing angle approximation does not work. In such cases, we treat the stellar radiation transfer by a $2 \mathrm{D}$ ray-tracing method. This modification is found to work in the present calculation. We assume dust consisting of spherical astronomical silicate grains (Draine \& Lee 1984) and the dust size distribution given by $n(a) \propto a^{-3.5}$, where $a$ is the grain radius (Mathis et al. 1977). As Perrin et al. (2009) discussed that the maximum grain size is about $1 \mu \mathrm{m}$, we set the minimum and maximum grain sizes to be $0.005 \mu \mathrm{m}$ and $2.5 \mu \mathrm{m}$, respectively. The stellar parameters used are: the effective temperature $\mathrm{T}_{*}$ $=9,500 \mathrm{~K}$, the radius $\mathrm{R}_{*}=2.5 \mathrm{R}_{\odot}$, and the mass $\mathrm{M}_{*}=2.4 \mathrm{M}_{\odot}$ (van Boekel et al. 2005). We assume a power-law surface density $\Sigma=\Sigma_{1}(r / \mathrm{AU})^{-p}$ between $r_{\text {in }}$ and $r_{\text {out }}$, where p=1 (Hartmann et al. 1998), $\Sigma_{1}=70 \mathrm{~g} \mathrm{~cm}^{-2}, r_{\text {in }}=0.5 \mathrm{AU}$ and $r_{\text {out }}=1000 \mathrm{AU}$. The surface density is the total (gas + dust) one and we also assume a gas-to-dust mass ratio of 100 . We chose $\Sigma_{1}$ and $r_{\text {out }}$ so as to reproduce the observed far-infrared to sub-mm/mm flux densities and $r_{\text {in }}$ is the radius where the dust temperature becomes about 1,400 K. In addition, we introduce the boundary radius $R_{c}$ that separates the inner and outer regions of the disk because the inner region (within $70-110 \mathrm{AU}$ of the central star) is reported to show a deficiency in the mm/sub-mm dust continuum emission (Piétu et al. 2005; Lin et al. 2006), which is attributed to a decrease in the surface density of dust grains smaller than mmsize in the inner region. To account for this information in our disk modeling, we consider that the surface density is reduced by a scaling factor $f_{c}$ of $0.1,0.01$, and 0.001 for $r<R_{c}$ compared to the power-law surface density of the outer disk. The explored parameter space is summarized in Table 1 . This reduction of the inner surface density makes the inner region much thinner than the outer disk, and as a result, a wall-like structure appears at the boundary radius. The upper part of the wall is directly illuminated by the central star; thus its temperature is enhanced like a surface super-heated layer (Chiang \& Goldreich 1997). On the other hand, the lower part of the wall remains cool because it is shadowed by the inner region (Mulders et al. 2010). In practice, we calculate the disk structures of the inner and outer regions separately, and connect them at the boundary radius $R_{c}$. The temperature of the illuminated upper part of the wall (i.e. inner edge of the outer region) is set to be the same as the surface layer at the wall. In addition, the same treatment is adopted at the most inner edge of the disk which is also directly illuminated by the central star. We need 2D radiative transfer calculations to be self-consistent (Mulders et al. 2010), which will be done in the future. 
On the basis of the derived disk structure, we calculate the model brightness distribution for various wavelengths and spectrum by a ray-tracing method along the observer's line of sight. The inclination angle is assumed to be $30^{\circ}$ (Fukagawa et al. 2004). To compare the model brightness distribution with our observation, we create a model image where the model brightness distribution is convolved with the observed PSF image shown in Fig. 1. Finally, we find that the best match with the observed radial profile is obtained with the scaling factor of the inner disk $f_{c}=0.01$ and the boundary radius $R_{c}=88 \mathrm{AU}$. This is comparable to the inner radius derived by Piétu et al. (2005) ( $\sim 110$ AU for dust and $\sim 70$ AU for CO). Fig. 2 shows the best-fit model brightness distribution at $25 \mu \mathrm{m}$, the model image for Subaru/COMICS and its peak-normalized radial profile. Structural parameters of the best-fit model such as the radial distributions of the surface density and height, are shown in Fig. 3. The surface height was determined by the height where the stellar Planck mean opacity became unity towards the central star. The spectrum of the best-fit model also matches with that of AB Aur from $10 \mu \mathrm{m}$ to $\sim 1 \mathrm{~mm}$ (Helou \& Walker 1988; Meeus et al. 2001; Acke et al. 2004, and references therein) as shown in Fig. 4. For wavelengths shorter than $10 \mu \mathrm{m}$, our model spectrum underestimates the observed spectrum of AB Aur. This may be due to the emission from the inner-most gas disk (Tannirkulam et al. 2008) or from the puffed-up inner rim (Natta et al. 2001), neither of which is considered in our model. The contribution of these inner structures to the $25 \mu \mathrm{m}$ flux density is negligible; the spectrum at 1-8 $\mu \mathrm{m}$ can be well fitted by an additional blackbody of temperature $1,400 \mathrm{~K}$ (the upper solid line) and this blackbody component emits $1.4 \mathrm{Jy}$ at $25 \mu \mathrm{m}$, only $3 \%$ of the $25 \mu \mathrm{m}$ Infrared Astronomical Satellite (IRAS) flux density of 48 Jy (see Fig. 4).

\section{Discussion}

\subsection{Comparison with other observations and validity of the wall-like structure}

A reduction of the surface density inside the boundary radius $\left(R_{c} \sim 88 \mathrm{AU}\right)$ and the resultant wall-like structure at $R_{c}$ can explain the extended emission we observed at $24.6 \mu \mathrm{m}$. Additionally, this interpretation is consistent with the $\mathrm{mm} /$ sub- $\mathrm{mm}$ interferometric observations of AB Aur. Furthermore, it can explain the properties of the AB Aur disk that appear more point-like at shorter MIR wavelengths. In order to determine the extent of the AB Aur disk at these shorter MIR wavelengths from our best-fit model, we calculated 11.4 and 18 $\mu \mathrm{m}$ model brightness distributions of this model, and convolved them with a gaussian model PSF comparable to the diffraction limit of an $8.2 \mathrm{~m}$ telescope. Then we measured FWHMs of the PSF convolved model images and derived gaussian FWHMs of 8 AU at $11.4 \mu \mathrm{m}$ and $21 \mathrm{AU}$ at $18 \mu \mathrm{m}$ by using the quadratic subtraction method. The value at $18 \mu \mathrm{m}$ is con- 
sistent with previous observational results by Mariñas et al. (2006) and that at $11.4 \mu \mathrm{m}$ is slightly narrower than the observations (Mariñas et al. 2006), but the general trend is fine. The more compact nature of the AB Aur disk at the shorter MIR wavelengths relative to $25 \mu \mathrm{m}$ is due to not only the radial temperature distribution but also the temperature of the wall-like structure $(96 \mathrm{~K})$. This temperature is too low for dust to radiate efficiently at shorter wavelengths, resulting in little change of the appearance of the disk at shorter wavelengths. A similar situation is also seen in another Herbig Ae/Be star HD 142527, which is only marginally extended at $10 \mu \mathrm{m}$, but clearly extended at $24.5 \mu \mathrm{m}$ (Fuijwara et al. 2006).

Another piece of collateral evidence for the wall-like structure at $\sim 88 \mathrm{AU}$ is the agreement between the temperature $(93 \mathrm{~K})$ of the blackbody component derived from the spectrum of AB Aur (Meeus et al. 2001) and the temperature (96K) of the wall at $88 \mathrm{AU}$ in our bestfit disk model. Meeus et al. (2001) suggested that this temperature might reflect the dust temperature at the onset of disk flaring. Their suggestion is qualitatively similar to ours in the sense that the wall in our model can be interpreted as the onset of the outer disk flaring.

Furthermore, the annulus of dust at $\sim 100 \mathrm{AU}$ is observed in the NIR polarimetric coronagraphic images (Oppenheimer et al. 2008; Perrin et al. 2009; Hashimoto et al. 2010). Inside the dust annulus of the NIR scattered light, the gap is reported at a radius of $0.5 " \sim 0.6 "$ (72 86AU; Hashimoto et al. 2010). This also supports the onset of the disk flaring and the presence of a wall-like structure at $\sim 88$ AU in the AB Aur disk.

\subsection{The AB Aur disk: a pre-transitional disk?}

Combined with the relatively young age of the central star and the pristine environment as indicated by the global envelope structure, AB Aur has been thought to be a prototypical example of newly formed Herbig Ae/Be star (e.g. Grady et al. 1999). However, the inner region of the $\mathrm{AB}$ Aur disk possesses little gas and dust as revealed by mm/sub-mm interferometric observations (Piétu et al. 2005; Lin et al. 2006), indicating the evolved nature of the disk. Such an apparently paradoxical situation may have led to the difficulty faced by Chen \& Jura (2003) in explaining the AB Aur properties with a simple flared disk model. A reduction in the inner disk surface density such as that used in our model can solve this problem. This situation is qualitatively similar to pre-transitional disks introduced for some $\mathrm{T}$ Tauri stars (Espaillat et al. 2007) in the sense that an inner disk separated from the optically thick outer disk is present. We propose that the AB Aur disk is also a pre-transitional disk.

Various mechanisms for the clearing of the inner region have been suggested such as 
dust coagulation (e.g. Dullemond \& Dominik 2005), inside-out evacuation by the magnetrotational instability (e.g. Chiang \& Murray-Clay 2007; Suzuki et al. 2009), photoevaporation (e.g. Alexander et al. 2006), dynamical effects of companions (e.g. Varnière et al. 2006a, b), and so on. In the case of AB Aur, it is not clear which mechanism is the most plausible; however, the presence of planetary companions has been suggested, which may have caused the inner hole (e.g. Piétu et al. 2005; Jang-Condell \& Kuchner 2010; Hashimoto et al. 2010). The pre-transitional disk nature of the AB Aur disk may reflect ongoing planet formation.

We are grateful to all of the staff members of the Subaru Telescope for providing us the opportunities to perform these observations and for their support. This research was partially supported by KAKENHI (Grant-in-Aid for Young Scientists B: 21740141) by the Ministry of Education, Culture, Sports, Science and Technology (MEXT) of Japan.

\section{REFERENCES}

Acke, B., van den Ancker, M. E., Dullemond, C. P., van Boekel, R., \& Waters, L. B. F. M. 2004, A\&A, 422, 621

Alexander, R. D., Clarke, C. J., \& Pringle, J. E. 2006, MNRAS, 369, 229

Benisty, M., Tatulli, E., Ménard, F., \& Swain, M. R. 2010, A\&A, 511, A75

Bouwman, J., de Koter, A., Dominik, C., \& Waters, L. B. F. M. 2003, A\&A, 401, 577

Cardelli, J. A., Clayton, G. C., \& Mathis, J. S. 1989, ApJ, 345, 245

Chiang, E. I., \& Goldreich, P. 1997, ApJ, 490, 368

Chiang, E., \& Murray-Clay, R. 2007, Nature Physics, 3, 604

Chen, C. H., \& Jura, M. 2003, ApJ, 591, 267

Corder, S., Eisner, J., \& Sargent, A. 2005, ApJ, 622, L133

DeWarf, L. E., Sepinsky, J. F., Guinan, E. F., Ribas, I., \& Nadalin, I. 2003, ApJ, 590, 357

Dominik, C., Dullemond, C. P., Waters, L. B. F. M., \& Walch, S. 2003, A\&A, 398, 607

Draine, B. T., \& Lee, H. M. 1984, ApJ, 285, 89

Dullemond, C. P., van Zadelhoff, G. J., \& Natta, A. 2002, A\&A, 389, 464 
Dullemond, C. P., \& Dominik, C. 2005, A\&A, 434, 971

Engelke, C. W., Price, S. D., \& Kraemer, K. E. 2006, AJ, 132, 1445

Espaillat, C., Calvet, N., D’Alessio, P., Hernández, J., Qi, C., Hartmann, L., Furlan, E., \& Watson, D. M. 2007, ApJ, 670, L135

Fujiwara, H., et al. 2006, ApJ, 644, L133

Fukagawa, M., et al. 2004, ApJ, 605, L53

Fukagawa, M., Tamura, M., Itoh, Y., Kudo, T., Imaeda, Y., Oasa, Y., Hayashi, S. S., \& Hayashi, M. 2006, ApJ, 636, L153

Grady, C. A., Woodgate, B., Bruhweiler, F. C., Boggess, A., Plait, P., Lindler, D. J., Clampin, M., \& Kalas, P. 1999, ApJ, 523, L151

Grady, C. A., et al. 2009, ApJ, 699, 1822

Hartmann, L., Calvet, N., Gullbring, E., \& D’Alessio, P. 1998, ApJ, 495, 385

Hashimoto, J., et al. 2010, in preparation

Helou, G., \& Walker, D. W. 1988, Infrared astronomical satellite (IRAS) catalogs and atlases. Volume 7, p.1-265, 7,

Jang-Condell, H., \& Kuchner, M. J. 2010, ApJ, 714, L142

Kataza, H., Okamoto, Y., Takubo, S., Onaka, T., Sako, S., Nakamura, K., Miyata, T., \& Yamashita, T. 2000, Proc. SPIE, 4008, 1144

Leinert, C., et al. 2004, A\&A, 423, 537

Lin, S.-Y., Ohashi, N., Lim, J., Ho, P. T. P., Fukagawa, M., \& Tamura, M. 2006, ApJ, 645, 1297

Liu, W. M., Hinz, P. M., Hoffmann, W. F., Brusa, G., Miller, D., \& Kenworthy, M. A. 2005, ApJ, 618, L133

Liu, W. M., Hinz, P. M., Meyer, M. R., Mamajek, E. E., Hoffmann, W. F., Brusa, G., Miller, D., \& Kenworthy, M. A. 2007, ApJ, 658, 1164

Mariñas, N., Telesco, C. M., Fisher, R. S., Packham, C., \& Radomski, J. T. 2006, ApJ, 653, 1353 
Mathis, J. S., Rumpl, W., \& Nordsieck, K. H. 1977, ApJ, 217, 425

Meeus, G., Waters, L. B. F. M., Bouwman, J., van den Ancker, M. E., Waelkens, C., \& Malfait, K. 2001, A\&A, 365, 476

Mulders, G. D., Dominik, C., \& Min, M. 2010, arXiv:1001.2146

Natta, A., Prusti, T., Neri, R., Wooden, D., Grinin, V. P., \& Mannings, V. 2001, A\&A, 371, 186

Okamoto, Y. K., Kataza, H., Yamashita, T., Miyata, T., Sako, S., Takubo, S., Honda, M., \& Onaka, T. 2003, Proc. SPIE, 4841, 169

Oppenheimer, B. R., et al. 2008, ApJ, 679, 1574

Pantin, E., Bouwman, J., \& Lagage, P. O. 2005, A\&A, 437, 525

Perrin, M. D., Schneider, G., Duchene, G., Pinte, C., Grady, C. A., Wisniewski, J. P., \& Hines, D. C. 2009, ApJ, 707, L132

Piétu, V., Guilloteau, S., \& Dutrey, A. 2005, A\&A, 443, 945

Sako, S., et al. 2003, PASP, 115, 1407

Suzuki, T. K., Muto, T., \& Inutsuka, S.-i. 2009, arXiv:0911.0311

Tannirkulam, A., et al. 2008, ApJ, 689, 513

van Boekel, R., Min, M., Waters, L. B. F. M., de Koter, A., Dominik, C., van den Ancker, M. E., \& Bouwman, J. 2005, A\&A, 437, 189

van den Ancker, M. E., The, P. S., Tjin A Djie, H. R. E., Catala, C., de Winter, D., Blondel, P. F. C., \& Waters, L. B. F. M. 1997, A\&A, 324, L33

Varnière, P., Bjorkman, J. E., Frank, A., Quillen, A. C., Carciofi, A. C., Whitney, B. A., \& Wood, K. 2006, ApJ, 637, L125

Varnière, P., Blackman, E. G., Frank, A., \& Quillen, A. C. 2006, ApJ, 640, 1110

This preprint was prepared with the AAS IATEX macros v5.2. 


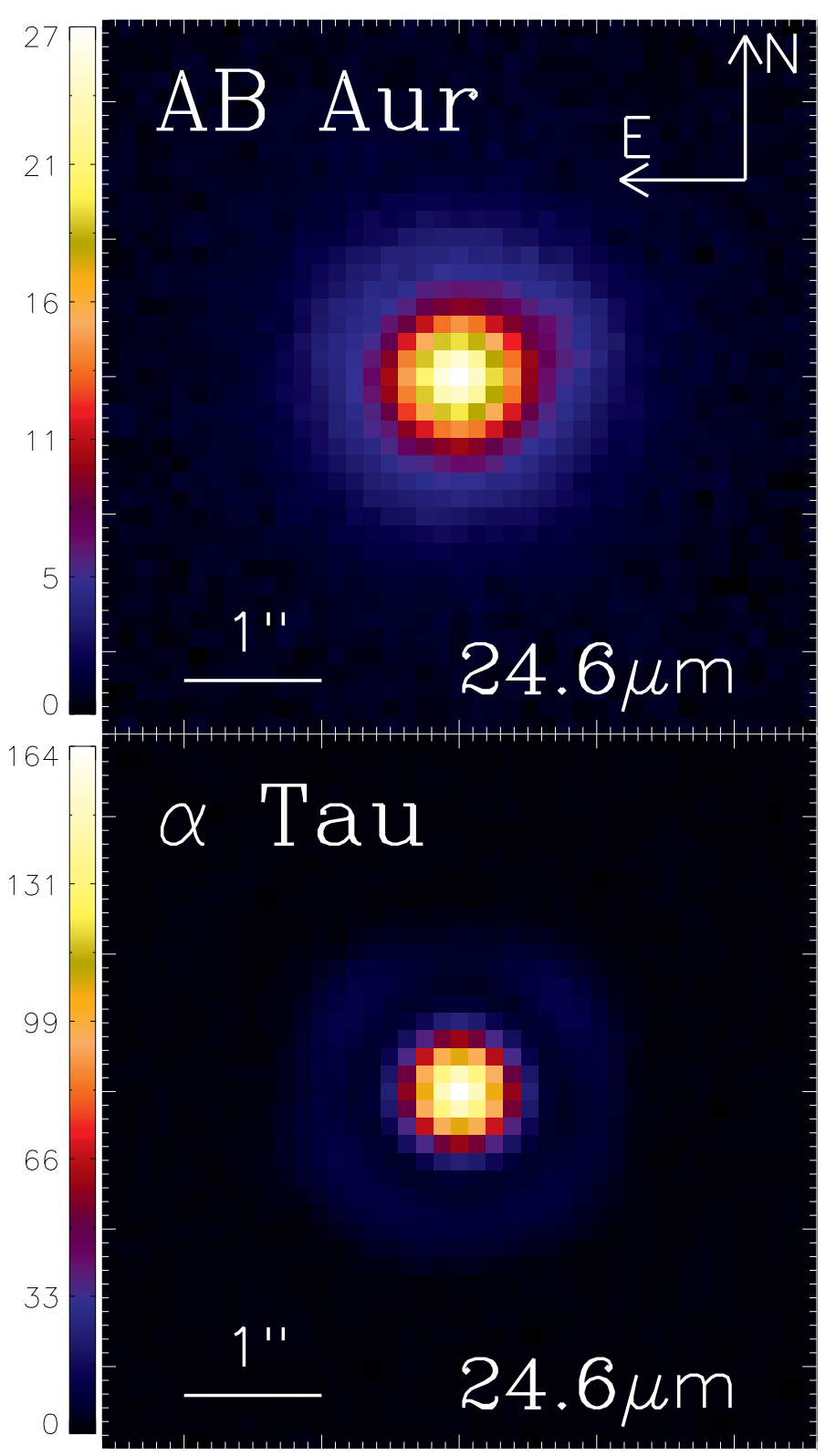

Fig. 1. - Shift-and-added image of AB Aur (top) and $\alpha$ Tau as a PSF reference (bottom) at $24.6 \mu \mathrm{m}$. The surface brightness is given in Jy/ $\operatorname{arcsec}^{2}$ and scaled from 0 to the peak value of each image. North is up and east is to the left for the AB Aur image; however, the PSF image is aligned to match the same instrument rotation angle as the AB Aur image for a similar optical configuration. 
Table 1. Range of Parameters Explored in Model Grid

\begin{tabular}{cc}
\hline \hline$\Sigma$ scaling factor $f_{c}$ & Boundary radius $R_{c}[\mathrm{AU}]$ \\
\hline 0.1 & $75,81,88,95,102,110,119,128$ \\
0.01 & $65,70,75,81,88,95,102,110,119$ \\
0.001 & $33,38,44,52,60,65,70,75,81,88,95,102$ \\
\hline \hline
\end{tabular}

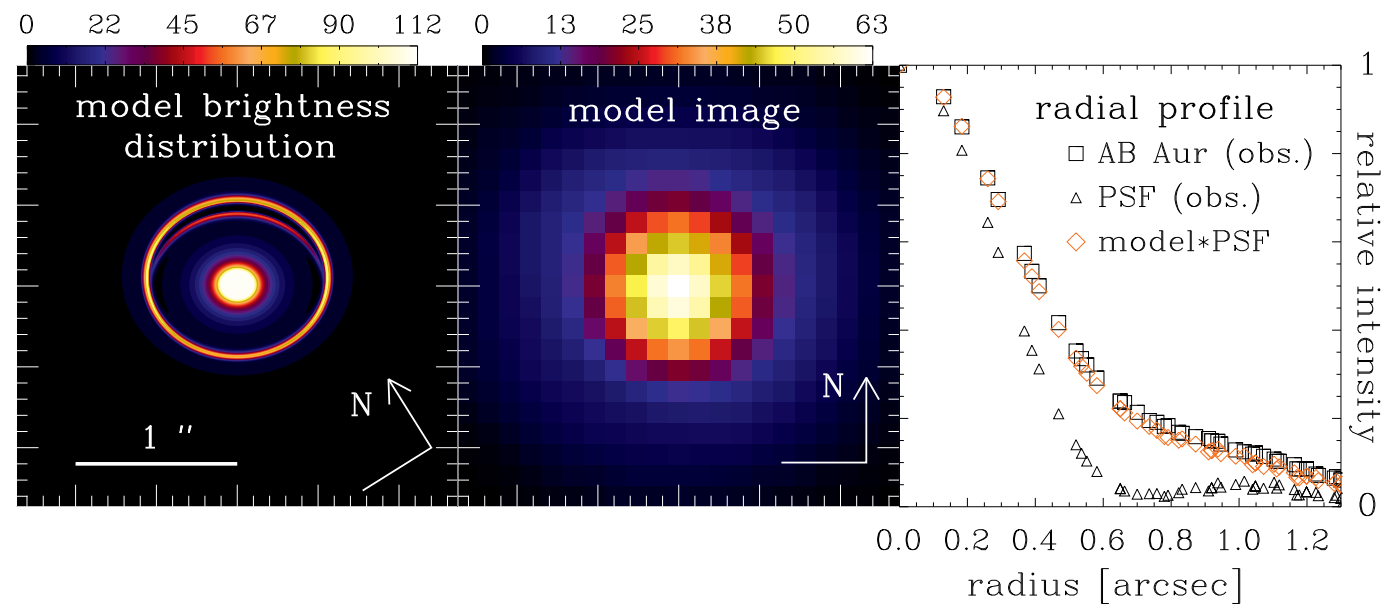

Fig. 2.- Plots comparing the observations and the best-fit model (boundary radius $R_{c}=88$ $\mathrm{AU}$ and scaling factor $f_{c}=0.01$ ). The left panel represents the model brightness distribution at $25 \mu \mathrm{m}$ in $\mathrm{Jy} / \operatorname{arcsec}^{2}$. The middle panel shows the model image where the model brightness distribution is binned to the COMICS plate-scale $(0.13$ " / pixel) and convolved with the observed PSF shown in Fig.1; additionally, the image was rotated so that the disk majoraxis position-angle was $58^{\circ}$ (Fukagawa et al. 2004). The right panel shows peak-normalized azimuthally-averaged radial profile plots of the observed AB Aur image (squares), the PSF image (triangles), and the binned model image convolved with the PSF shown in the middle panel (diamonds); horizontal axis represents radius in arcseconds. 

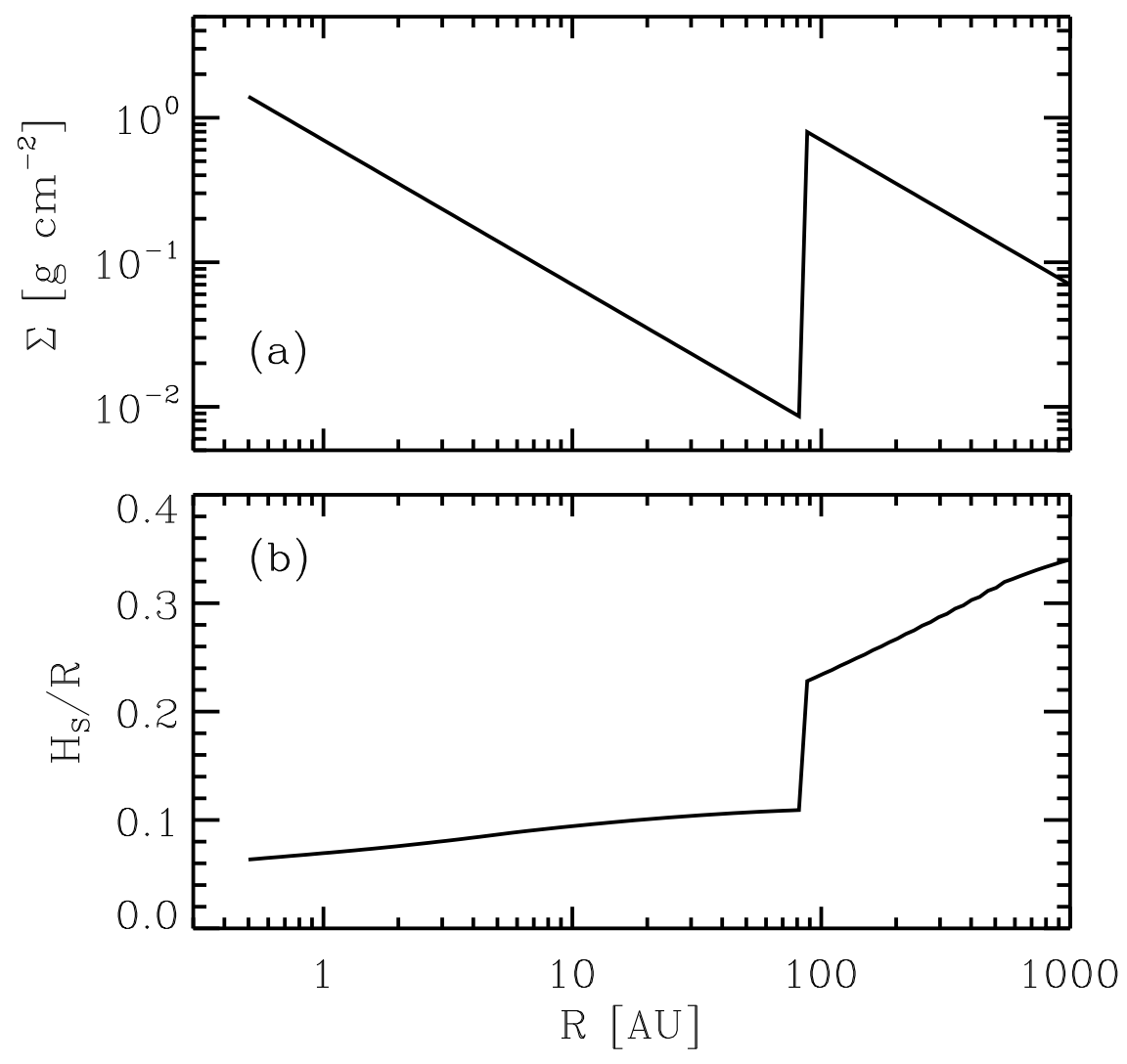

Fig. 3.- (a) Radial distribution of the summed gas and dust surface density for the best-fit model (boundary radius $R_{c}=88 \mathrm{AU}$ and scaling factor $f_{c}=0.01$ ). (b) Radial distribution of the surface height $H_{s}$ normalized by $\mathrm{R}$ for the best-fit model. The surface height was determined by the height where the stellar Planck mean opacity became unity along the line of sight to the central star. A wall-like structure is present at the boundary radius $R_{c}$. 


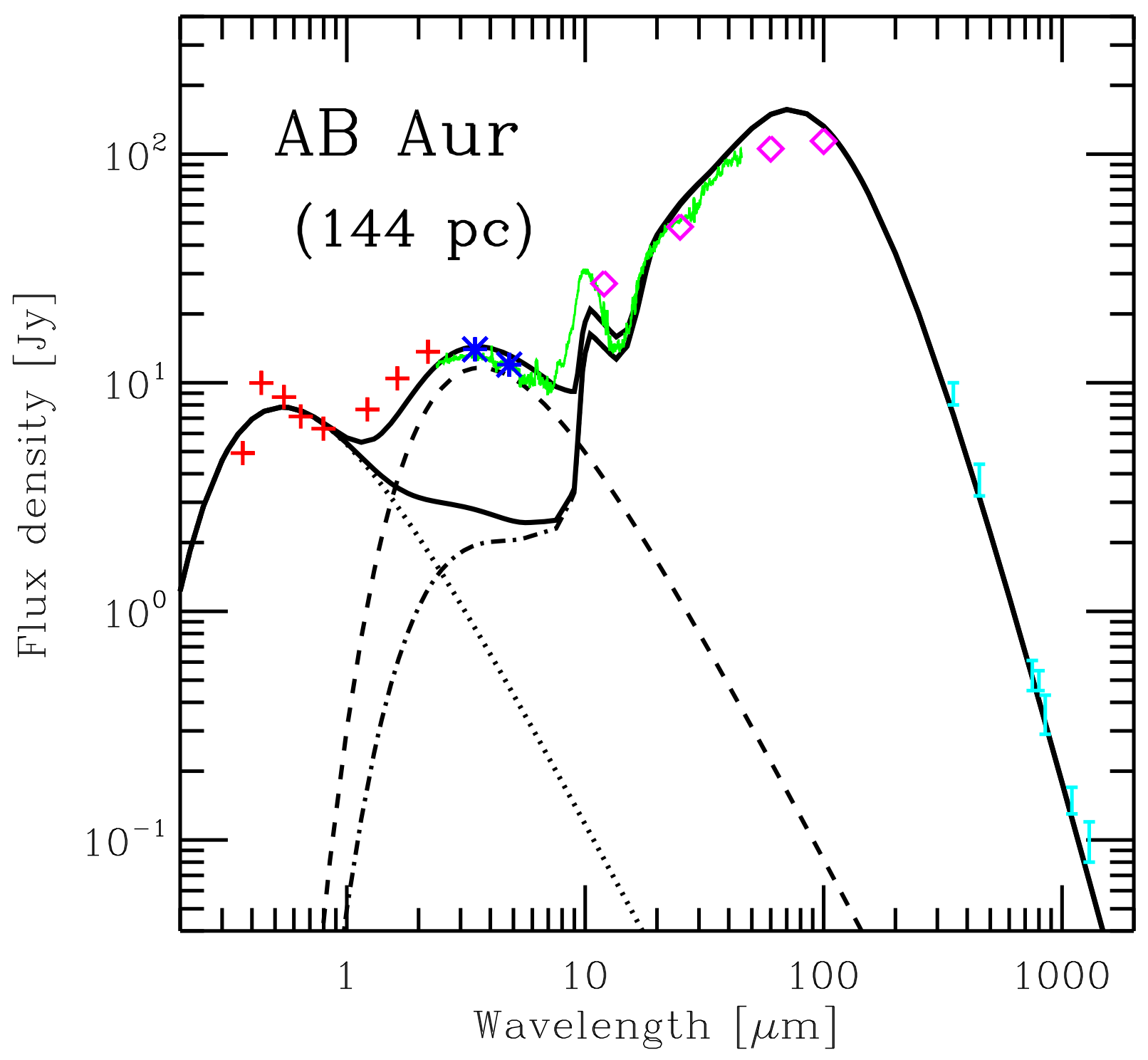

Fig. 4. - Spectrum of AB Aur observed and that of our best-fit model. Observed data are from Helou \& Walker (1988); Meeus et al. (2001); Acke et al. (2004) and references therein. Reddening correction is applied using $A v=0.5 \mathrm{mag}$ (Acke et al. 2004) assuming the extinction law of Cardelli et al. (1989). The stellar emission is shown in dotted line. The dot-dashed line represents emission from the dust disk of our best-fit model. The dashed line is for the additional 1400K blackbody needed to fit the observed spectrum, which is not considered in our best-fit model. The lower solid line is a sum of the emission from the star and the dust disk, while the upper solid line is a sum of the star, disk and 1400K blackbody emission. 